\title{
Effect of Annealing Temperature on the Electric and Dielectric Properties of $\mathrm{Se}_{70} \mathrm{Te}_{15} \mathrm{Bi}_{15}$ Films
}

\author{
H.E. ATYiA* \\ Physics Department, Faculty of Education, Ain Shams University, Roxy, Cairo, Egypt \\ and \\ Physics Department, Faculty of Science, Taif University, Kingdom of Saudi Arabia \\ (Received April 10, 2013; in final form October 24, 2013)
}

\begin{abstract}
The effect of annealing at different temperatures between $T_{\mathrm{g}}$ and $T_{\mathrm{c}}$ on the $\mathrm{AC}$ conductivity and dielectric properties was studied for $\mathrm{Se}_{70} \mathrm{Te}_{15} \mathrm{Bi}_{15}$ films grown by thermal evaporation technique. The films were characterized by X-ray diffraction, differential thermal analysis, and energy dispersive X-ray spectroscopy. X-ray diffraction analysis shows the occurrence of amorphous to polycrystalline transformation for films annealed at annealing temperature $T_{\mathrm{a}} \geq 473 \mathrm{~K}$. AC conductivity $\sigma_{\mathrm{AC}}(\omega)$ was studied as a function of $T_{\mathrm{a}}$, frequencies $(0.1-100 \mathrm{kHz})$ and working temperatures $(303-393 \mathrm{~K})$. It was found that $\sigma_{\mathrm{AC}}(\omega)$ obeyed $A \omega^{s}$ law. According to the values of $s$ and its temperature dependence, the AC conduction mechanism was determined in terms of the correlated barrier hopping and quantum mechanical tunneling models for the as deposited and annealed films, respectively. The DC and AC activation energies were determined as a function of $T_{\mathrm{a}}$. Values of dielectric constant $\varepsilon_{1}$ and dielectric loss $\varepsilon_{2}$ were found to increase with increasing $T_{\mathrm{a}}$. A Debye-like relaxation of dielectric behavior was observed for polycrystalline films, and was found to be a thermally activated process.
\end{abstract}

DOI: 10.12693/APhysPolA.125.98

PACS 72.80.Ng, 77.22.-d

\section{Introduction}

Great attention has been given to chalcogenide glasses in recent year, mainly due to their wide range of applications in solid state devices both in scientific and technological fields $[1,2]$. Se is one of the most important chalcogen elements which has applications in electrophotography. The pure Se has some disadvantage due to its short life time and low sensitivity, which can be improved by incorporating elements such as Te. Se-Te alloys are widely used for various applications in many fields as optical recording media and electrographical applications. The composition $\mathrm{Se}_{85} \mathrm{Te}_{15}$ has been studied by several authors [3-5] because of its ease of fabrication in the bulk a thin film forms. The addition of impurities like $\mathrm{Bi}$ is particularly of much interest as it has produced a change in optical, electrical and thermal properties of $\mathrm{Se}-\mathrm{Te}$ chalcogenide glasses [6-8].

A lot of work has been done on ternary chalcogenide glasses having $\mathrm{Se}-\mathrm{Te}-\mathrm{Bi}$ composition [9-11]. Determination of $\mathrm{AC}$ conductivity mechanism of materials is very important, which represents an experimental method to obtain information about the existence and location of localized states in chalcogenide glasses.

There are various models which have been proposed to make clear the conduction mechanisms in amorphous semiconductors. These models are quantum-mechanical tunneling (QMT) model [12, 13], small polaron tunneling model (SPTM) [14, 15], large polaron tunnel-

*e-mail: hebaelghrip@hotmail.com ing model (LPTM) [14] and correlated barrier hopping (CBH) model [16-18]. Dielectric analysis measurements give information about the electrical characteristic of materials such as the insulating nature which represents its ability to store electrical charge and the conductive nature, which represents its ability to transfer electrical charge [19] in addition to the study of the dielectric relaxation which is very important to understand the nature of dielectric losses in the material. The thermal processes are known to be important in inducing crystallization in semiconducting chalcogenide glasses.

The aims of this work are: (1) study of the effect of heat treatment on the structure, electric and dielectric properties for thermal evaporated $\mathrm{Se}_{70} \mathrm{Te}_{15} \mathrm{Bi}_{15}$ films. (2) Study of the working temperature and frequency dependence of $\mathrm{AC}$ conductivity, dielectric constant and dielectric loss for as-deposited and annealed $\mathrm{Se}_{70} \mathrm{Te}_{15} \mathrm{Bi}_{15}$ films. (3) Discussion of the obtained results according to different models of $\mathrm{AC}$ conductivity for chalcogenide glasses.

\section{Experimental techniques}

Glassy alloy of $\mathrm{Se}_{70} \mathrm{Te}_{15} \mathrm{Bi}_{15}$ was prepared by quenching technique. High purity $(99.999 \%)$ materials are weighted according to their atomic percentage, and sealed in dry silica tube under a vacuum of $10^{-5} \mathrm{~Pa}$. The ampoule is placed in the stable zone of an oscillatory furnace. The ampoule temperature was raised from that of the room temperature slowly at a rate of $3-4 \mathrm{~K} / \mathrm{min}$ to $1273 \mathrm{~K}[8]$ and held at this temperature for $15 \mathrm{~h}$, during heating, the ampoule was constantly rocked to obtain homogeneous glassy alloy. Then molten material was 
quenched in an icy-water. Films with different thicknesses of $\mathrm{Se}_{70} \mathrm{Te}_{15} \mathrm{Bi}_{15}$ were obtained by thermal evaporation under vacuum $2 \times 10^{-5} \mathrm{~Pa}$ from bulk material onto clean glass substrates. The substrate temperature was held at that of room temperature (below $50^{\circ} \mathrm{C}$ ) during the deposition process. Film thickness was measured by Tolansky's interferometric method [20]. The chemical composition of the investigated samples was checked by energy dispersive X-ray (EDX) analysis using a (JEOL 5400) scanning electron microscope. X-ray diffraction (XRD) analysis was used to investigate the structure of the composition in thin film form. The structural identification was confirmed also by differential thermal analysis (DTA) thermogram. For ac measurements films were sandwiched between two aluminum electrodes of a suitable thickness $(500 \mathrm{~nm})$. A programmable automatic RLC bridge (PM 6304Phillips) was used to measure the sample impedance $Z$, the sample capacitance $C$ and loss $\operatorname{tangent} \tan \delta$ directly. All investigated samples were represented on the screen of the bridge by a resistance $R$ connected in parallel with capacitance $C$. The total conductivity $\sigma_{\text {tot }}(\omega)$ is given by $\sigma_{\text {tot }}(\omega)=\frac{1}{Z} \frac{L}{A}$, where $L$ is the sample thickness and $A$ is the sample cross-section area. The dielectric constant $\varepsilon_{1}$ was calculated by applying the relation $\varepsilon_{1}=\frac{C}{\varepsilon_{0}} \frac{L}{A}$, where $\varepsilon_{0}$ is the permittivity of free space $\left(\varepsilon_{0}=8.85 \times 10^{-12} \mathrm{~F} / \mathrm{m}\right)$. The dielectric loss $\varepsilon_{2}$ was calculated from the relation $\varepsilon_{2}=\varepsilon_{1} \tan \delta$ where $\delta=90-\phi, \phi$ is the phase angle. Film samples were annealed at different temperature (423 and $473 \mathrm{~K}$ ) for $2 \mathrm{~h}$. The temperature of the sample was recorded by means of digital multimeter (Protec 81 ) provided by a chromelalumel thermocouple adjacent to the sample.

\section{Results and discussion}

\subsection{Structural investigation}

X-ray diffraction patterns of as-deposited $\mathrm{Se}_{70} \mathrm{Te}_{15} \mathrm{Bi}_{15}$ films showed that the as-deposited films are of amorphous nature as shown in Fig. 1a for film thickness $380 \mathrm{~nm}$ as a representative example. To investigate the effect of annealing temperatures on the structure of $\mathrm{Se}_{70} \mathrm{Te}_{15} \mathrm{Bi}_{15}$ films. Different thicknesses were annealed for $2 \mathrm{~h}$ at 423 and $473 \mathrm{~K}$, Fig. 1b,c shows X-ray diffraction patterns of the annealed films. The absence of sharp structural peaks for annealed films at annealing temperature $T_{\mathrm{a}}=423 \mathrm{~K}$ confirms the amorphous nature while the presence of sharp structural peaks for annealed films at $T_{\mathrm{a}}=473 \mathrm{~K}$ confirms the polycrystalline nature of these annealed films. The same results were obtained for all thicknesses in the range $688-219 \mathrm{~nm}$.

DTA was carried out at a constant rate of $10 \mathrm{~K} / \mathrm{min}$, which was used for measuring the glass transition and crystallization temperatures for bulk samples. The obtained glass transition and crystallization temperatures are 123 and $210^{\circ} \mathrm{C}$. It must be noticed that the annealing temperatures were taken in between the glass transition and crystallization temperatures of the prepared samples.

EDX spectrum analysis using a scanning electron microscope (JEOL 5400) of as-deposited and annealed $\mathrm{Se}_{70} \mathrm{Te}_{15} \mathrm{Bi}_{15}$ films have shown that the composition of

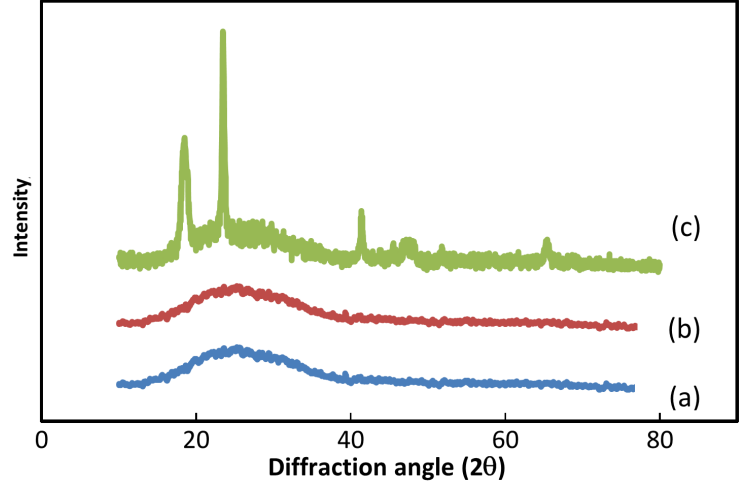

Fig. 1. X-ray diffraction patterns for $(a)$ as-deposited, (b) $T_{\mathrm{a}}=423 \mathrm{~K}$, (c) $T_{\mathrm{a}}=473 \mathrm{~K} \mathrm{Se}_{70} \mathrm{Te}_{15} \mathrm{Bi}_{15}$ films of thickness $380 \mathrm{~nm}$.

films near $\mathrm{Se}_{70} \mathrm{Te}_{15} \mathrm{Bi}_{15}$ as shown in Table I, which indicated that the annealing process of films does not affect their composition. Figure 2 shows the EDX spectrum for the as-deposited $\mathrm{Se}_{70} \mathrm{Te}_{15} \mathrm{Bi}_{15}$ film of thickness $219 \mathrm{~nm}$ as a representative example.

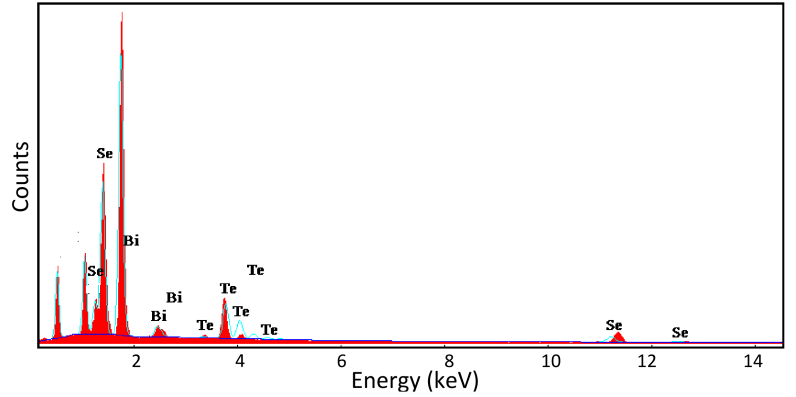

Fig. 2. EDX spectrum for as-deposited $\mathrm{Se}_{70} \mathrm{Te}_{15} \mathrm{Bi}_{15}$ film of thickness $219 \mathrm{~nm}$.

Elemental analysis of $\mathrm{Se}_{70} \mathrm{Te}_{15} \mathrm{Bi}_{15}$ (at.\%).

TABLE I

\begin{tabular}{c|c|c|c}
\hline \hline Element & $\begin{array}{c}\text { As-deposited } \\
\text { films }\end{array}$ & $\begin{array}{c}\text { Annealed films } \\
\text { at } T_{\mathrm{a}}=423 \mathrm{~K}\end{array}$ & $\begin{array}{c}\text { Annealed films } \\
\text { at } T_{\mathrm{a}}=473 \mathrm{~K}\end{array}$ \\
\hline $\mathrm{Se}$ & 71.05 & 70.37 & 71.53 \\
$\mathrm{Te}$ & 14.61 & 14.72 & 14.06 \\
$\mathrm{Bi}$ & 14.34 & 14.91 & 14.41 \\
Total & 100 & 100 & 100
\end{tabular}

\subsection{Frequency and temperature dependence of $A C$ conductivity}

AC conductivity $\sigma_{\mathrm{AC}}(\omega)$ is related to the total conductivity $\sigma_{\text {tot }}(\omega)$, and DC conductivity $\sigma_{\mathrm{DC}}$ according to the relation [21]:

$$
\sigma_{\mathrm{AC}}(\omega)=\sigma_{\text {tot }}(\omega)-\sigma_{\mathrm{DC}}
$$

It was found that the measured $\sigma_{\text {tot }}(\omega)$ increases by about 3-4 orders of magnitude of $\sigma_{\mathrm{DC}}$. Thus, the mea- 
sured values of $\sigma_{\text {tot }}(\omega)$ in obtained results will be considered the typical values of $\sigma_{\mathrm{AC}}(\omega)$.

The AC conductivity $\sigma_{\mathrm{AC}}(\omega)$ increases with frequency according to the following equation [21]:

$$
\sigma_{\mathrm{AC}}(\omega)=A \omega^{s},
$$

where $s$ is the frequency exponent, $A$ is a constant and $\omega$ is the angular frequency.

The variation of $\sigma_{\mathrm{AC}}(\omega)$ with frequency in the range 0.1-100 kHz was studied, for as-deposited and annealed $\mathrm{Se}_{70} \mathrm{Te}_{15} \mathrm{Bi}_{15}$ films $\left(T_{\mathrm{a}}=423\right.$ and $\left.473 \mathrm{~K}\right)$ at different working temperatures $(303-393 \mathrm{~K})$. Figure $3 \mathrm{a}-\mathrm{c}$ depicts the frequency dependence of $\sigma_{\mathrm{AC}}(\omega)$ at different working temperature for the as deposited and annealed films with similarly the same thickness $(445 \mathrm{~nm})$ as a representative example. It can be inferred that at any working temperature $\sigma_{\mathrm{AC}}(\omega)$ increases linearly with increasing frequency for all films with different $T_{\mathrm{a}}$. Values of the frequency exponent $s$ were estimated from the slopes of straight lines in Fig. 3. The calculated values of $s$ were found decreased with increasing temperature $(1>s>0.6)$ for pre-crystalline films, as shown in Fig. 4, and temperature independent with a nearly constant value $(s \approx 0.81)$ for polycrystalline films.

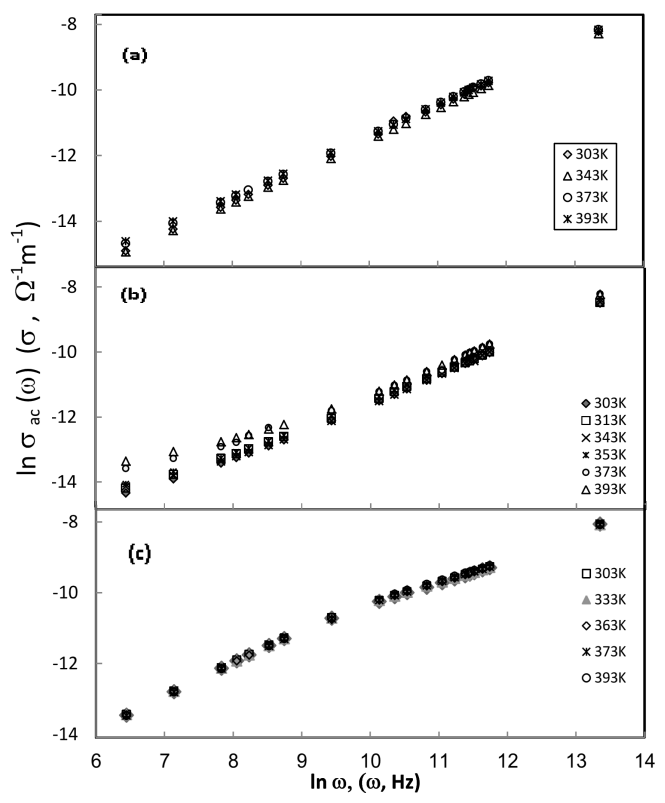

Fig. 3. Frequency dependence of the ac conductivity $\sigma_{\mathrm{ac}}(\omega)$ for (a) as-deposited, (b) $T_{\mathrm{a}}=423 \mathrm{~K}$, (c) $T_{\mathrm{a}}=$ $473 \mathrm{~K} \mathrm{Se} 70 \mathrm{Te}_{15} \mathrm{Bi}_{15}$ films of nearly the same thickness $(445 \mathrm{~nm})$ at different temperatures.

The room temperature $\sigma_{\mathrm{AC}}(\omega)$ was drawn vs. $\ln \omega$ for as-deposited and annealed $\mathrm{Se}_{70} \mathrm{Te}_{15} \mathrm{Bi}_{15}$ films at different $T_{\mathrm{a}}$ in Fig. 5. It is observed from this figure that $\sigma_{\mathrm{AC}}(\omega)$ at any frequency increases with increasing annealing temperature. The same results were obtained when this relation was studied at elevated working temperatures.

Different models have been proposed to interpret the $\mathrm{AC}$ electrical conduction depending on the values of the

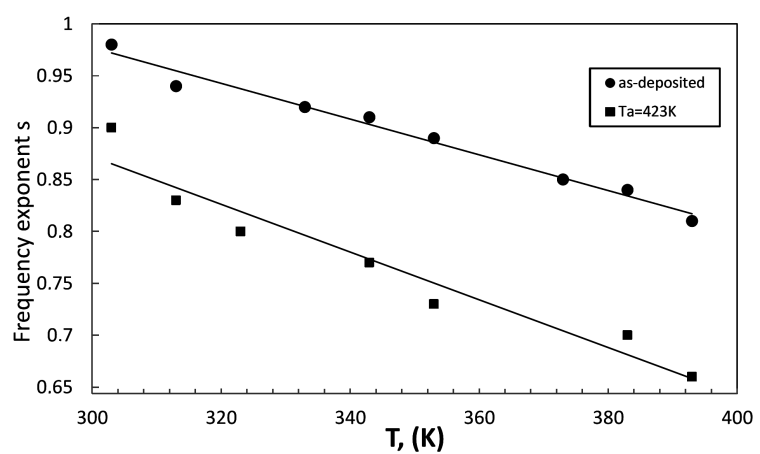

Fig. 4. Temperature dependence of the frequency exponent $s$ for pre-crystalline $\mathrm{Se}_{70} \mathrm{Te}_{15} \mathrm{Bi}_{15}$ films.

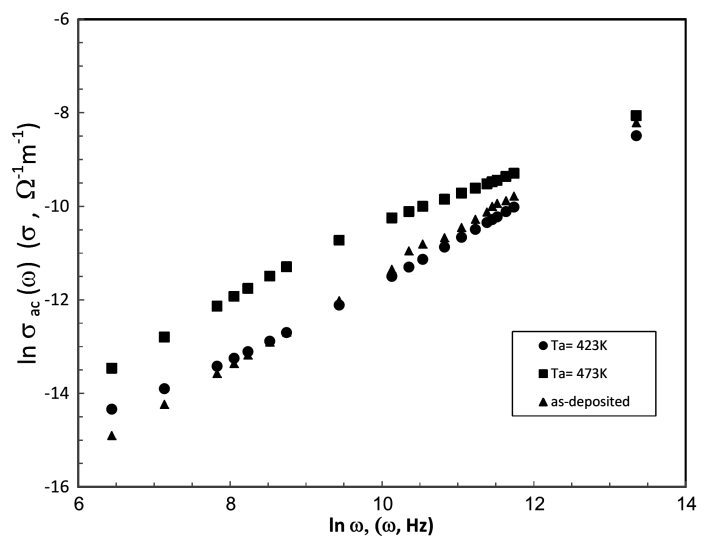

Fig. 5. Frequency dependence of room temperature $\sigma_{\mathrm{ac}}(\omega)$ for as-deposited and annealed $\mathrm{Se}_{70} \mathrm{Te}_{15} \mathrm{Bi}_{15}$ films.

frequency exponent $s$ and its temperature dependence. The CBH model proposed by Elliott [16] has been applied to the chalcogenide glasses semiconductors. According to this model, values of the frequency exponent $s$ ranged from $0.7-1$ at room temperature and decreased with increasing temperatures. This model is in good agreement with the obtained results for pre-crystalline $\mathrm{Se}_{70} \mathrm{Te}_{15} \mathrm{Bi}_{15}$ films (as-deposited and annealed films at $T_{\mathrm{a}}=423 \mathrm{~K}$ ). Therefore the frequency dependence of $\sigma_{\mathrm{AC}}(\omega)$ for pre-crystalline $\mathrm{Se}_{70} \mathrm{Te}_{15} \mathrm{Bi}_{15}$ films can be explained in terms of $\mathrm{CBH}$ model. According to $\mathrm{CBH}$ model the conduction occurs via a bipolaron hopping process where two polarons simultaneously hop over the potential barrier between two charged defect states $D^{+}$and $D^{-}$. The barrier height is correlated with the inter-site separation via a Coulombic interaction. Hence the exponent $s$ obeying the following equation [22, 23]:

$$
s=1-\frac{6 k T}{W_{\mathrm{M}}-k T \ln \left(\frac{1}{\omega \tau_{0}}\right)},
$$

where $W_{\mathrm{M}}$ is the maximum barrier height of infinite separation which is called the energy polaron binding, $k$ is the Boltzmann constant, $T$ is absolute temperature and $\tau_{0}$ - characteristic relaxation time which is in the order 
of atom vibrational periodic $\approx 10^{-13} \mathrm{~s}$. For large value of $\frac{W_{\mathrm{M}}}{k T}, \mathrm{Eq}$. (3) can be rewritten as

$$
s=1-\frac{6 k T}{W_{\mathrm{M}}} \text {. }
$$

For neighboring sites of separation $R$, the Coulomb wells overlap, resulting in the lowering of the effective barrier from $W_{\mathrm{M}}$ to value $W_{\mathrm{h}}$, which for the case of a single electron transition is given by

$$
W_{\mathrm{h}}=W_{\mathrm{M}}-\frac{e^{2}}{\pi \varepsilon_{0} \varepsilon_{1} R},
$$

where the hopping distance $R$ is given by the following equation:

$$
R=\frac{e^{2}}{\pi \varepsilon_{0} \varepsilon_{1}\left[W_{\mathrm{M}}-k T \ln \left(\frac{1}{\omega \tau_{0}}\right)\right]},
$$

and the lower bound (cut-off) to hopping distance becomes

$$
R_{\text {min }}=\frac{e^{2}}{\pi \varepsilon_{0} \varepsilon_{1} W_{\mathrm{M}}},
$$

where $e$ is the electron charge, $\varepsilon_{1}$ - the dielectric constant and $\varepsilon_{0}$ - the permittivity of air. The calculated values of $W_{\mathrm{M}}$ and $R_{\min }$ parameters are 0.296 and $0.230 \mathrm{eV}$, and $3.24 \times 10^{-10}$ and $2.78 \times 10^{-10} \mathrm{~m}$ for as-deposited and annealed $\left(T_{\mathrm{a}}=423 \mathrm{~K}\right) \mathrm{Se}_{70} \mathrm{Te}_{15} \mathrm{Bi}_{15}$ films, respectively.

Another model was suggested by Austin and Mott [24], who assumed that conduction occurs by means of a thermally assisted QMT model [25]. According to this model the exponent $s$ is given by the following relation [26]:

$$
s=1-\frac{4}{\ln \left(\frac{v_{0}}{\omega}\right)},
$$

where $s$ is almost equal to 0.81 independent of temperature. Therefore, QMT model is considered applicable to the obtained results for polycrystalline $\mathrm{Se}_{70} \mathrm{Te}_{15} \mathrm{Bi}_{15}$ films.

The working temperature dependence of $\sigma_{\mathrm{AC}}(\omega)$ was studied for as-deposited and annealed $\mathrm{Se}_{70} \mathrm{Te}_{15} \mathrm{Bi}_{15}$ films at different frequencies. Figure $6 \mathrm{a}-\mathrm{c}$ depicts the plot of $\ln \sigma_{\mathrm{AC}}(\omega)$ vs. $1000 / T$ for as-deposited and annealed $\mathrm{Se}_{70} \mathrm{Te}_{15} \mathrm{Bi}_{15}$ films at different frequencies. It can be inferred from these figures that $\sigma_{\mathrm{AC}}(\omega)$ was increased linearly with the reciprocal of temperature with one slope giving single small values of activation energy dependence of frequency. To clarify the temperature dependence of $\sigma_{\mathrm{AC}}(\omega)$, Fig. $6 \mathrm{~d}$ shows the plot of $\ln \sigma_{\mathrm{AC}}(\omega)$ vs. $1000 / T$ for films at $0.1 \mathrm{kHz}$ as a representative example. This mechanism is due to hopping between states near the mobility edges where the density of states is not constant. The same results are obtained for other chalcogenide compositions [27]. The above relation is different from the nonlinear relation obtained for some compositions $[28,19]$.

The obtained results suggested that $\sigma_{\mathrm{AC}}(\omega)$ is a thermally activated process from different localized states in the gap or its tails using the well-known equation

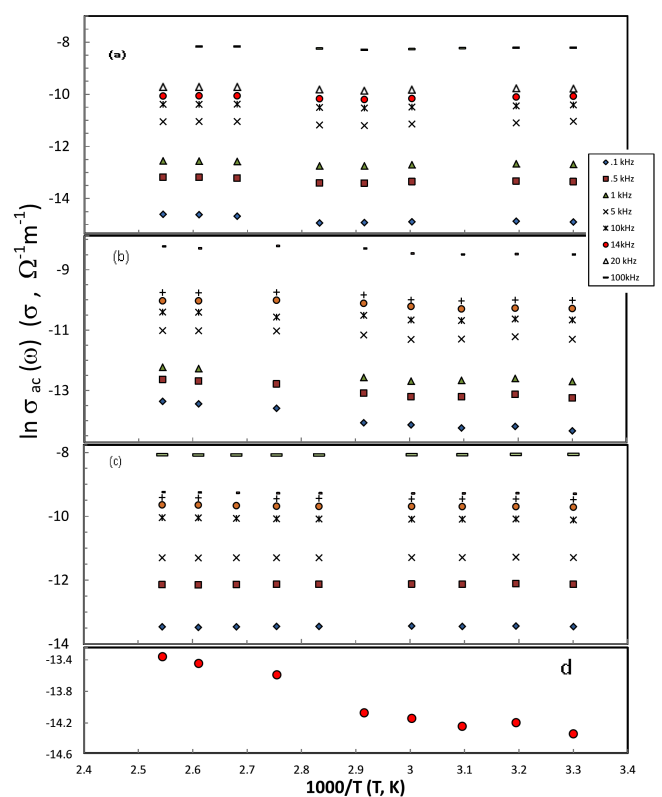

Fig. 6. Temperature dependence of the ac conductivity $\sigma_{\mathrm{ac}}(\omega)$ (a) as-deposited, (b) $T_{\mathrm{a}}=423 \mathrm{~K}$, (c) $T_{\mathrm{a}}=$ $473 \mathrm{~K}$, (d) as-deposited at $0.1 \mathrm{kHz}$, for $\mathrm{Se}_{70} \mathrm{Te}_{15} \mathrm{Bi}_{15}$ films of nearly the same thickness $(445 \mathrm{~nm})$ at different frequencies.

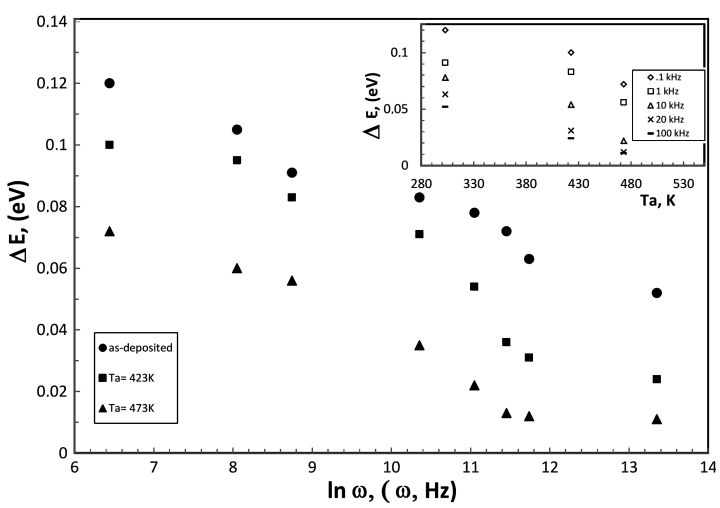

Fig. 7. Frequency dependence of $\Delta E_{\sigma}(\omega)$ for as-deposited and annealed $\mathrm{Se}_{70} \mathrm{Te}_{15} \mathrm{Bi}_{15}$ films. Inset figure shows the annealing temperature dependence of $\Delta E_{\sigma}(\omega)$ at different frequencies.

$$
\sigma_{\mathrm{AC}}(\omega)=\sigma_{0} \exp \left(\frac{-\Delta E_{\sigma}(\omega)}{k T}\right),
$$

where $\sigma_{0}$ is pre-exponential factor and $\Delta E_{\sigma}(\omega)$ is the $\mathrm{AC}$ electrical conduction activation energy. The calculated values of $\Delta E_{\sigma}(\omega)$ were $0.11 \mathrm{eV}>\Delta E_{\sigma}(\omega)>0.052 \mathrm{eV}, 0.108 \mathrm{eV}>\Delta E_{\sigma}(\omega)>0.03 \mathrm{eV}$ and $0.072 \mathrm{eV}>\Delta E_{\sigma}(\omega)>0.01 \mathrm{eV}$ for as-deposited annealed $\mathrm{Se}_{70} \mathrm{Te}_{15} \mathrm{Bi}_{15}$ films at $T_{\mathrm{a}}=423$ and $473 \mathrm{~K}$, respectively. The lower values of $\Delta E_{\sigma}(\omega)$ indicate that the electric conduction takes place by hopping of charge carriers between the defect states near the Fermi level. Values of DC electrical conduction activation energy $\Delta E_{\sigma}(\mathrm{DC})$ 
can be obtained by extrapolation of the frequency dependence of $\Delta E_{\sigma}(\omega)$ to zero frequency. Values of $\Delta E_{\sigma}(\omega), \Delta E_{\sigma}(\mathrm{DC})$ and $\ln \sigma_{0}$ were listed in Table II for as-deposited and annealed $\mathrm{Se}_{70} \mathrm{Te}_{15} \mathrm{Bi}_{15}$ films.

The frequency dependence of $\Delta E_{\sigma}(\omega)$ for the investi- gated films is shown in Fig. 7. It is clear that $\Delta E_{\sigma}(\omega)$ decreases with increasing frequency as well as increasing $T_{\mathrm{a}}$ as shown in inset of Fig. 7. This may be attributed to the increase of applied field frequency which enhances the electronic jump between localized states [27].

TABLE II

Values of the activation energy and pre exponent factor for as-deposited and annealed $\mathrm{Se}_{70} \mathrm{Te}_{15} \mathrm{Bi}_{15}$ films at different $T_{\mathrm{a}}$.

\begin{tabular}{c|c|c|c|c|c|c}
\hline \hline \multirow{2}{*}{$\begin{array}{c}\text { Frequency } \\
{[\mathrm{kHz}]}\end{array}$} & \multicolumn{2}{|c|}{ As-deposited films } & \multicolumn{2}{c|}{ Films annealed at $T_{\mathrm{a}}=423 \mathrm{~K}$} & \multicolumn{2}{c}{ Films annealed at $T_{\mathrm{a}}=473 \mathrm{~K}$} \\
\cline { 2 - 7 } & $\Delta E_{\sigma}[\mathrm{eV}]$ & $\ln \sigma_{0}\left(\sigma_{0}\left[\Omega^{-1} \mathrm{~m}^{-1}\right]\right)$ & $\Delta E_{\sigma}[\mathrm{eV}]$ & $\ln \sigma_{0}\left(\sigma_{0}\left[\Omega^{-1} \mathrm{~m}^{-1}\right]\right)$ & $\Delta E_{\sigma}[\mathrm{eV}]$ & $\ln \sigma_{0}\left(\sigma_{0}\left[\Omega^{-1} \mathrm{~m}^{-1}\right]\right)$ \\
\hline 0 & 0.196 & & 0.188 & & 0.14 & -13.1 \\
0.1 & 0.11 & -12.5 & 0.108 & -10.5 & 0.072 & -11.4 \\
0.5 & 0.105 & -11.9 & 0.095 & -10.2 & 0.06 & -10.8 \\
1 & 0.091 & -11.21 & 0.086 & -10.1 & 0.056 & -9.8 \\
5 & 0.083 & -9.95 & 0.071 & -9.7 & 0.035 & -9.5 \\
10 & 0.078 & -9.4 & 0.054 & -9.1 & 0.022 & -9.38 \\
15 & 0.072 & -9.3 & 0.036 & -8.8 & 0.013 & -9.17 \\
20 & 0.063 & -8.95 & 0.031 & -8.7 & 0.012 & -8.1 \\
100 & 0.052 & -7.6 & 0.024 & -7.39 & 0.011 & \\
\end{tabular}

The same behavior was obtained for $\Delta E_{\sigma}(\mathrm{DC})$ with annealing temperature as shown in Table II. The increase in the electrical conductivity and consequently, a decrease in the activation energy with thermal annealing above $T_{\mathrm{g}}$ can be interpreted by assuming the production of surface dangling bonds around crystallites [29]. Heat treatment causes the crystallites to breakdown into smaller crystals increasing the number of surface dangling bonds. As the number of dangling bonds and defects increase, the concentration of localized states increases with an increase in annealing temperature decreasing the activation energy. The discrepancy between $\mathrm{DC}$ and $\mathrm{AC}$ activation energy is expected since the charge carriers in the DC conduction would choose the easiest paths which include the same large jumps, while this is not so important with ac conduction [30].

\subsection{Frequency and temperature dependence of the dielectric constant $\varepsilon_{1}$}

Figure 8a illustrates the variation of the dielectric constant $\varepsilon_{1}$ with frequency at different working temperatures for as-deposited $\mathrm{Se}_{70} \mathrm{Te}_{15} \mathrm{Bi}_{15}$ films. One can observe that, in general $\varepsilon_{1}$ decreases with increasing frequencies, at low frequencies, the dielectric constant $\varepsilon_{1}$ shows dispersive behavior and rises rapidly as a working temperature increases, a strong dispersion is also observed at frequency below $1 \mathrm{kHz}$. This can be attributed to the fact that as the frequency increases, the variation in the field becomes too rapid for the molecular dipoles to follow, so that their contribution to the polarization becomes less with a measurable lag because of internal orientional forces [31]. It is clear also that at a constant frequency $\varepsilon_{1}$ increases with increasing working temperature. This behavior can be attributed to the fact that the orientional polarization is connected with the thermal motion of molecules, so the dipoles cannot orient themselves at low temperature. If the temperature increases, the orientation of dipoles is facilitated and this increases the values of orientational polarization increasing the dielectric constant $\varepsilon_{1}[32]$.

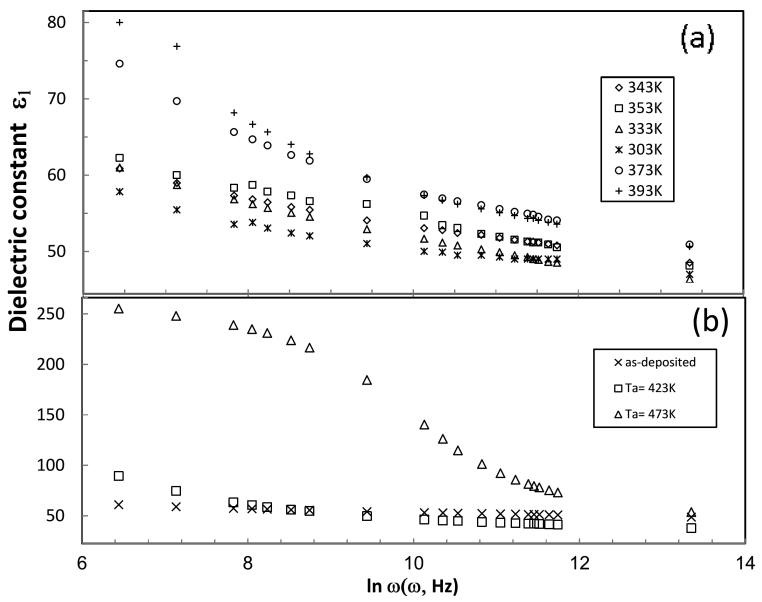

Fig. 8. (a) Frequency dependence of the dielectric constant $\varepsilon_{1}$ for as-deposited $\mathrm{Se}_{70} \mathrm{Te}_{15} \mathrm{Bi}_{15}$ films at different working temperatures. (b) Frequency dependence of room temperature dielectric constant $\varepsilon_{1}$ for as-deposited and annealed $\mathrm{Se}_{70} \mathrm{Te}_{15} \mathrm{Bi}_{15}$ films.

Figure $8 \mathrm{~b}$ shows the frequency dependence of the $\varepsilon_{1}$ measured at room working temperature as a representa- 
tive example for as-deposited and annealed $\mathrm{Se}_{70} \mathrm{Te}_{15} \mathrm{Bi}_{15}$ films. It is clear that for any frequency $\varepsilon_{1}$ increases with increasing annealing temperature. The same result was obtained at evaluated working temperature.

\subsection{Frequency and temperature dependence of the dielectric loss $\varepsilon_{2}$}

Figure $9 \mathrm{a}$ and $\mathrm{b}$ shows the frequency dependence of dielectric loss $\varepsilon_{2}$ for pre-crystalline $\mathrm{Se}_{70} \mathrm{Te}_{15} \mathrm{Bi}_{15}$ films at different working temperatures for similar thickness $445 \mathrm{~nm}$ as a representative example. It is clear that $\varepsilon_{2}$ decreases with increasing frequency, which can be attributed to the fact that the migration of ions in the glass is the main source of the dielectric loss at low frequencies. Accordingly, the dielectric loss at low and moderate frequencies is characterized by high values of $\varepsilon_{2}$ due to the contribution of ion jump and conduction loss of ion migration, in addition to the ion polarization loss. However, at high frequencies, the ion vibrations may be the only source of dielectric loss [27]. It is clear also that at any frequency $\varepsilon_{2}$ increases with increasing working temperatures, which can be interpreted by Stevels [33], who divided the relaxation phenomenon into three parts, conduction losses, dipole losses and vibrational losses. At low temperatures conduction losses have minimum value, as the temperature increases the electrical conduction losses increase, which increases the values of $\varepsilon_{2}$ with increasing temperature. The frequency dependence of $\varepsilon_{2}$ for polycrystalline $\mathrm{Se}_{70} \mathrm{Te}_{15} \mathrm{Bi}_{15}$ films annealed at $T_{\mathrm{a}}=473 \mathrm{~K}$ is shown in Fig. 10 at different working temperatures. Peaks in the $\varepsilon_{2}$ were observed at given frequencies indicating the characteristic feature of Debye-type relaxation process and as the temperature increases the peak shifts to higher frequency. These curves observed in Fig. 10 were called the Debye curves and they are symmetrical around $\omega_{\mathrm{m}}$, where $\omega_{\mathrm{m}}$ is the angular frequency corresponding to maximum observation, $\omega_{\mathrm{m}}=2 \pi f_{\max }$, where $f_{\max }$ is the relaxation frequency which is given by the following formula [34]:

$$
f_{\max }=1 / 2 \pi \tau
$$

where $\tau$ is the relaxation time.

Figure 11 shows the temperature dependence of the relaxation time $\tau$ as $\ln \tau$ vs. $1000 / T$ according to the following equation [35]:

$$
\tau=\tau_{\infty} \exp \left(\frac{E_{0}}{k T}\right),
$$

where $\tau_{\infty}$ is the relaxation time at finite temperature and $E_{0}$ is the activation energy for relaxation. The calculated values of $\tau_{\infty}$ and $E_{0}$ are $8.38 \times 10^{-6} \mathrm{~s}$ and $0.058 \mathrm{eV}$, respectively. One can observe that the Debye type relaxation did not appear in as-deposited and annealed $\mathrm{Se}_{70} \mathrm{Te}_{15} \mathrm{Bi}_{15}$ films at $T_{\mathrm{a}}=423 \mathrm{~K}$ (precrystalline films), while it was observed for annealed $\mathrm{Se}_{70} \mathrm{Te}_{15} \mathrm{Bi}_{15}$ films at $T_{\mathrm{a}}=473 \mathrm{~K}$ (polycrystalline films). This behavior may be attributed according to Guintini et al. [36] to dielectric loss in chalcogenide glasses. This model is based on Elliott's idea [16] of hopping of charge carriers over a potential barrier between charged defect states $\left(D^{-}\right.$and $\left.D^{+}\right)$.

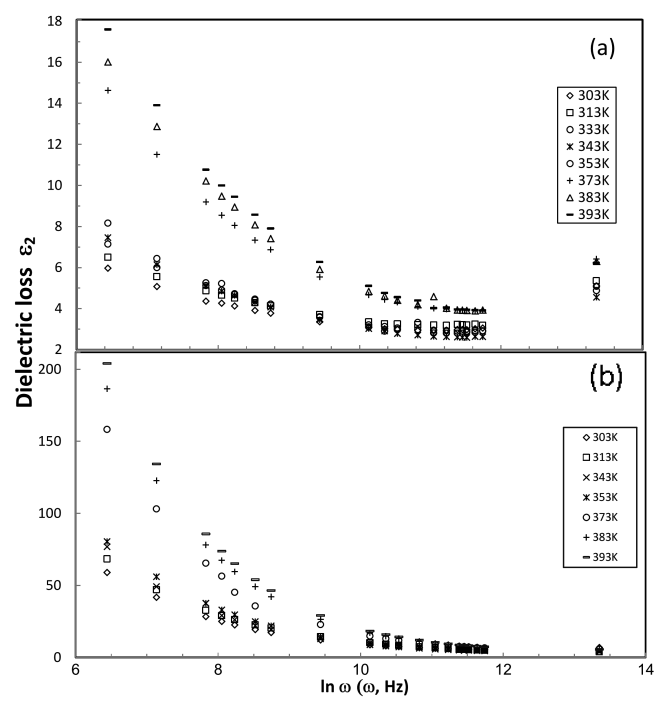

Fig. 9. Frequency dependence of the dielectric loss $\varepsilon_{2}$ for (a) as-deposited films, (b) annealed films at $T_{\mathrm{a}}=$ $423 \mathrm{~K}$ of nearly the same thickness $445 \mathrm{~nm}$ at different temperatures.

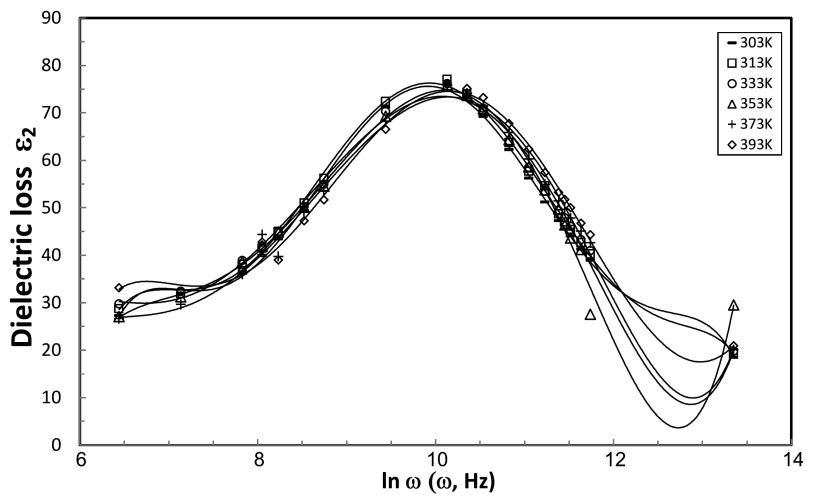

Fig. 10. Frequency dependence of the dielectric loss $\varepsilon_{2}$ for annealed $\mathrm{Se}_{70} \mathrm{Te}_{15} \mathrm{Bi}_{15}$ films $\left(T_{\mathrm{a}}=473 \mathrm{~K}\right)$ at different temperatures.

Each pair forms dipoles with a relaxation time depending on activation energy [37]. More dipoles in a studied films can be obtained with increase of the defects of states [38], which occurs as a result of annealing process. During this process the unsaturated bonds annealed out [39] producing many saturated bonds increasing the value

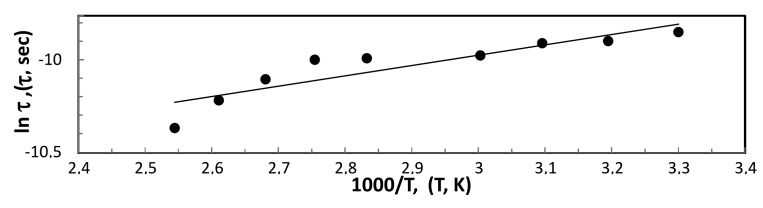

Fig. 11. The temperature dependence of the relaxation time $\tau$ for annealed $\mathrm{Se}_{70} \mathrm{Te}_{15} \mathrm{Bi}_{15}$ films at $T_{\mathrm{a}}=473 \mathrm{~K}$. 
of density of states for annealed films. According to the above explanation the change of $\sigma_{\mathrm{ac}}(\omega), \varepsilon_{1}, \varepsilon_{2}, s, \Delta E(\omega)$ for as-deposited and annealed $\mathrm{Se}_{70} \mathrm{Te}_{15} \mathrm{Bi}_{15}$ films can be discussed.

\section{Conclusion}

Amorphous films of $\mathrm{Se}_{70} \mathrm{Te}_{15} \mathrm{Bi}_{15}$ were prepared by thermal evaporation technique, then annealed at different annealing temperatures $T_{\mathrm{a}}$ to obtain an amorphous to polycrystalline transformation at $T_{\mathrm{a}} \geq 473 \mathrm{~K}$. Frequency and working temperature dependence of $\sigma_{\mathrm{ac}}(\omega)$ were studied for as-deposited and annealed films. The temperature dependence of the frequency exponent $s$ confirmed the applicability of $\mathrm{CBH}$ model for amorphous films and QMT model for polycrystalline films. DC and $\mathrm{AC}$ activation energies were found to be decreased with increase of annealing temperature. Both dielectric constant and dielectric loss increase with increasing working as well as annealing temperature and decrease with increasing frequency. A Debye-like relaxation in the dielectric loss was observed for polycrystalline films with a peak at maximum frequency $\omega_{m}$ shifted to higher frequency with increasing temperatures in the Debye curves. The temperature dependence of relaxation time was studied to determined the activation energy for relaxation $E_{0}$. The change of $\sigma_{\mathrm{ac}}(\omega), \varepsilon_{1}, \varepsilon_{2}, s, \Delta E(\omega)$ as a function of $T_{\mathrm{a}}$ can be explained according to increase of the density of localized states during annealing process.

\section{References}

[1] A.B. Seddon, J. Non-Cryst. Solids 184, 44 (1995).

[2] M.I. Lyas, M. Zulfequar, Z.H. Khan, M. Husain, Physica B 254, 57 (1998).

[3] N. Sharma, S. Kumar, Turk. J. Phys. 31, 161 (2007).

[4] V.S. Kushwaha, A. Kumar, J. Mater. Sci. 42, 2712 (2007).

[5] V. Sharma, J. Optoelectron. Adv. Mater. 8, 1823 (2006).

[6] Mainika, S. Pankaj, S.C. Katyal, T. Nagesh, J. Non-oxide Glasses 1, 90 (2009).

[7] A. Sharma, P.B. Barman, J. Thermal Anal. Calar. 96, 413 (2009).

[8] A. Sharma, P.B. Barman, Physica B 404, 1591 (2009).

[9] X.A. Fan, J.Y. Yang, W. Zhu, H.S. Yun, R.G. Chen, S.Q. Bao, X.K. Duan, J. Alloys Comp. 420, 256 (2006).

[10] D. Bourgault, C.G. Garampan, N. Carbone, J.A. Aymami, Thin Solid Films 516, 8579 (2008).
[11] Z. Abdel-Khalek Ali, G.H. Abel, A.S. Abd-rbo, Chalcog. Lett. 6, 125 (2009).

[12] M. Pollak, Philos. Mag. 23, 519 (1971).

[13] A. Ghosh, Phys. Rev. B 42, 5665 (1990).

[14] M. Pollak, G.E. Pike, Phys. Rev. Lett. 28, 1449 (1972).

[15] H.X. Lecleac, J. Phys. 40, 27 (1979).

[16] S.R. Elliot, Philos. Mag. B 36, 1291 (1977).

[17] S.R. Elliot, Philos. Mag. B 37, 553 (1978).

[18] K. Shimakawa, Philos. Mag. B 46, 123 (1982).

[19] H.E. Atyia, A.M. Farid, N.A. Hegab, Physica B 403 , 3980 (2008).

[20] S. Tolansky, Multiple-Beam Interference Microscopy of Metals, Academic Press, London 1970, p. 55.

[21] A.K. Jonscher, Nature 267, 673 (1977).

[22] G.E. Pike, Phys. Rev. B 6, 1572 (1972).

[23] S.R. Elliott, Adv. Phys. 36, 135 (1987).

[24] I.G. Austin, N.F. Mott, Adv. Phys. 18, 41 (1969).

[25] A. Ghosh, Phys. Rev. B 41, 1479 (1990).

[26] M. Pollak, T.H. Geball, Phys, Rev, B 122, 1742 (1961).

[27] N.A. Hegab, M.A. Afifi, H.E. Atyia, M.I. Ismael, Acta Phys. Pol. A 119, 416 (2011).

[28] M.A. Afifi, M.M. El-Nahass, A.E. Bekheet, I.T. Zedan, Physica B 400, 248 (2007).

[29] S. Chaudhuri, S.K. Biswas, J. Non-Cryst. Solids 54 179 (1983).

[30] M.A.M. Seyam, A.E. Bekheet, A. Elfalaky, Eur. Phys. 16, 99 (2001).

[31] B. Taree, Physics of Dielectric Materials, Mir Publishers, Moscow 1975.

[32] D. Deger, K.B. Ulutas, Balkan Phys. Lett. 8, 48 (2000).

[33] J.M. Stevels, Handbuch der Physik, Ed. S. Flugge, Springer, Berlin 1957, p. 350.

[34] R.I. Mohamed, J. Phys. Chem. Solids 61, 1357 (2000).

[35] A.S. Riad, M.T. Korayem, T.G. Abdel Mlik, Physica B 270, 140 (1999).

[36] J.C. Guintini, J.V. Zanchetta, D. Jullien, R. Enolie, P. Houenou, J. Non Cryst. Solids 45, 57 (1981).

[37] A.E. Stearn, H. Eyring, J. Chem. Phys. 5, 113 (1937).

[38] R. Arora, A. Kumar, Physica 175, 381 (1991).

[39] S. Hasegawa, S. Yazalci, T. Shimizu, Solid State Commun 26, 407 (1978). 